\title{
Therapeutic role of meloxicam targeting secretory clusterin-mediated invasion in hepatocellular carcinoma cells
}

\author{
JINGTAO ZHONG ${ }^{1}$, XIAOMING YU ${ }^{2}$, XIAOFENG DONG ${ }^{3}$, HONG LU $^{4}$, WUYUAN ZHOU ${ }^{1}$, \\ LEI LI ${ }^{1}$, ZHONGCHAO LI ${ }^{1}$, PENGFEI SUN ${ }^{1}$ and XUETAO SHI ${ }^{1}$ \\ ${ }^{1}$ Department of Hepatobiliary Surgery, Shandong Cancer Hospital Affiliated to Shandong University, \\ Shandong Academy of Medical Science, Jinan, Shandong 250117; ${ }^{2}$ Department of Ophthalmology, \\ Shandong Jiaotong Hospital, Jinan, Shandong 250031; ${ }^{3}$ Department of Hepatobiliary Surgery, The People's \\ Hospital of Guangxi Zhuang Autonomous Region, Nanning, Guangxi 530021; ${ }^{4}$ Department of Radiology, \\ Shandong Cancer Hospital Affiliated to Shandong University, Jinan, Shandong 250117, P.R. China
}

Received June 29, 2017; Accepted February 28, 2018

DOI: $10.3892 / \mathrm{ol} .2018 .8186$

\begin{abstract}
Recurrence and metastasis are the two leading causes of poor prognosis in patients with hepatocellular carcinoma (HCC). Secreted clusterin (sCLU) is a stress-induced chaperone that is overexpressed in HCC. However, the precise molecular mechanisms of sCLU in HCC invasion and migration are largely unknown. In the present study, it was indicated that downregulation of sCLU significantly alleviated invasiveness whereas overexpression of sCLU notably enhanced the number of invasive cells via mediating the expression level of MMP-2 and E-cadherin in Bel-7402 and SMMC-7721 cells. Furthermore, as an important mediator of invasiveness, sCLU may be responsible for proliferation and invasion suppression induced by meloxicam (a selective inhibitor of cyclooxygenase-2) in HCC cells. The combination of meloxicam and CLU shRNA significantly decreased invasion in HCC cells in vitro. Furthermore, it was observed that overexpression of sCLU significantly potentiated expression of p-AKT and MMP-2. However, downregulation of sCLU by CLU shRNA alleviated the extent of p-AKT. These results suggest the targeting of sCLU may be a novel therapeutic strategy against invasion and migration in HCC.
\end{abstract}

\section{Introduction}

Hepatocellular carcinoma (HCC) is the sixth most common form of cancer worldwide and the third most frequent cause of cancer-related death $(1,2)$. Hepatitis B virus (HBV), hepatitis C

Correspondence to: Dr Jingtao Zhong or Professor Xuetao Shi, Department of Hepatobiliary Surgery, Shandong Cancer Hospital Affiliated to Shandong University, Shandong Academy of Medical Science, 440 Jiyan Road, Jinan, Shandong 250117, P.R. China

E-mail: jingtao2005@126.com

E-mail: shixuetao9@126.com

Key words: hepatocellular carcinoma, meloxicam, secretory clusterin, invasion, AKT virus (HCV), alcohol abuse, and nonalcoholic fatty liver disease are recognized as the major risk factors for hepatocarcinogenesis (3). In spite of improving diagnostic techniques, efficient therapies are limited. Currently, surgical resection is still the most effective treatment for $\mathrm{HCC}$ at an early stage. However, only $20 \%$ of patients with HCC are diagnosed in early stages, while more than $80 \%$ of HCC cases are diagnosed at an advanced stage with inoperable distant metastases (4). Half of patients present with an advanced stage of HCC which must receive systemic therapy without surgical treatment, but the effectiveness of agents such as sorafenib used in the systematic treatment of advanced HCC is relatively limited (5). The poor prognosis of HCC patients is mainly associated with the high rate of intrahepatic metastasis after treatment. Therefore, it is imperative to develop new therapeutic strategies for HCC treatment.

Cyclooxygenase (COX)-2, a rate-limiting enzyme in the synthesis of prostaglandin (PG), has been reported as an anti-tumor target. A large number of studies have demonstrated that COX-2 influences many aspects of cancer cells including viability, motility, survival, invasiveness, and apoptosis resistance (6-9). Meloxicam, as a selective inhibitor of cyclooxygenase-2 (COX-2), is widely used for anti-inflammation. Accumulating evidences have revealed that the COX-2 inhibitor exerts an anti-proliferative response in various cancers (10-12). Our previous studies also revealed that meloxicam inhibited proliferation and led to apoptosis of HCC cells (13-16). However, the exact mechanisms of the anti-cancer effects regulated by meloxicam remain unclear.

The cytoprotective chaperone protein, clusterin, was first isolated from ram rete testes fluid and is synthesized as a full-length cluster $(60 \mathrm{kDa})$ in the mitochondria. It encodes two isoforms with paradoxical activities: Nuclear clusterin (nCLU) and secretory clusterin (sCLU) which plays a crucial role in regulating various pathophysiological processes such as tissue remodeling, reproduction, lipid transport, complement regulation, and apoptosis (17). sCLU, starting as an approximately $60 \mathrm{kDa}$ precursor peptide, has been considered as an anti-apoptotic protein. Recently, several studies 
have reported that $\mathrm{sCLU}$ is associated with resistance to chemotherapy. Constitutive over-expression of sCLU has been reported to confer chemoresistance in cancer therapy, however, down-regulation of sCLU sensitized pancreatic cancer cells to gemcitabine chemotherapy (18). Our previous studies also demonstrated that sCLU contributes to oxaliplatin resistance by activating the Akt pathway in HCC (19) and down-regulating sCLU could enhance the sensitivity of HCC cells to gemcitabine by activating the intrinsic apoptosis pathway (20). However, the precise mechanisms of sCLU in the resistance of HCC to chemotherapy are largely unknown. Therefore, we hypothesized that suppression of sCLU could potentiate the meloxicam-induced cytotoxicity in HCC cells. In this study, we tried to explore the role of sCLU in meloxicam-induced cytotoxicity in human HCC cells.

\section{Materials and methods}

Cell lines and culture. Human HCC cell lines, Bel-7402 were obtained from the American Type Culture Collection (ATCC, Manassas, VA, USA), and SMMC-7721 cell lines were purchased from the Type Culture Collection Cell Bank, Chinese Academy of Science, Shanghai, China. The cells were cultured in RPMI-1640 medium (Gibco; Thermo Fisher Scientific, Inc., Waltham, MA, USA) containing $10 \%$ fetal bovine serum (FBS; Gibco; Thermo Fisher Scientific, Inc.), $100 \mathrm{U} / \mathrm{ml}$ penicillin, and $100 \mu \mathrm{g} / \mathrm{ml}$ streptomycin (Gibco; Thermo Fisher Scientific, Inc.) at $37^{\circ} \mathrm{C}$ in $95 \%$ air and $5 \% \mathrm{CO}_{2}$.

Reagents and antibodies. The meloxicam (Mel) was purchased from EMD Millipore (Billerica, MA, USA), dissolved in dimethylsulfoxide (DMSO; Sigma-Aldrich, St. Louis, MO, USA) at 10 or $50 \mathrm{mM}$ stock. The concentration of DMSO never exceeded $0.6 \%$ (v/v) and equal amounts of DMSO were added to control cells. MK-2206 (a special inhibitor of AKT) was obtained from Merck KGaA (Darmstadt, Germany). Antibodies against MMP-2 (cat. no. 4022), AKT (cat. no. 9272), and phosphorylated AKT (p-AKT) (Ser473) (cat.no. 9271) were purchased from Cell Signaling Technology, Inc., (Danvers, MA, USA). Antibodies against E-cadherin (ab15148) and GAPDH (ab37168) were obtained from Abcam (Cambridge, UK). The antibody for sCLU (sc-5289) was purchased from Santa Cruz Biotechnology, Inc., (Dallas, TX, USA).

Wound-healing scratch assay. Cell monolayers grown to confluence on 6-well plastic dishes were wounded by scratching with a pipette tip. The cells were cultured in the presence or absence of meloxicam $(80 \mu \mathrm{M})$ for $24 \mathrm{~h}$. The wounds were photographed (10x objective) at the indicated time points.

Cell invasion assays. Briefly, the cell invasion assay was performed by adding Matrigel basement matrix to the upper chamber of transwells. $1 \times 10^{5}$ cells, in $300 \mu \mathrm{l}$ of RPMI-1640 medium (with $1 \%$ FBS) containing meloxicam were seeded to the upper chamber of Transwells (Corning, New York, USA). The bottom wells of the chambers were filled with $500 \mu \mathrm{l}$ RPMI-1640 medium (with 10\% FBS). After 24 h of incubation, the chambers were fixed with $95 \%$ ethanol and then stained with $1 \%$ crystal violet.
Reverse-transcription polymerase chain reaction (RT-PCR). Total RNA was extracted from the cells using Trizol reagent (Invitrogen; Thermo Fisher Scientific, Inc.), and cDNA was synthesized by using a cDNA synthesis kit (Invitrogen; Thermo Fisher Scientific, Inc.). The primers targeting MMP-2 were (5'-TGACGGTAAGGACGGACTC-3'; 5'-ATACTT CACACGGACCACTTG-3'), E-cadherin (5'-TGCCCAGAA AATGAAAAAGG-3'; 5'-GGATGACAGCGTGAGAGA-3'), and GAPDH (5'-TTACTCCTTGGAGGCCATGTGGGC-3'; 5'-ACTGCCACCCAGAAGACTGTGGATGG-3'). Expression levels were normalized to GAPDH. All protocols were carried out according to the manufacturer's instructions. Real-time PCR was performed using MX3000P Real-time PCR systems (Agilent Technologies, Inc., Santa Clara, CA, USA). Experiments were performed in triplicate, and the data were calculated by $\Delta \Delta \mathrm{Cq}$ methods.

Cell viability and western blotting assays. These methods have been previously described $(14,15,21)$. Cell viability assays were performed by using the Cell Counting Kit-8 (CCK-8; Dojindo Molecular Technologies, Inc., Kumamoto, Japan). After different treatments, protein concentrations in cell extracts were determined (Bio-Rad Laboratories, Inc., Hercules, CA, USA), equal amounts of each sample were resolved in SDS-PAGE gels, then transferred to a polyvinylidene fluoride (PVDF) membrane (EMD Millipore), and probed with the primary antibodies specific for sCLU, MMP-2, E-cadherin, AKT, and p-AKT.

sCLU-shRNA and pCDNA3.1-sCLU Transfection. Four potentially effective targets of sCLU were designed, synthesized, and inserted into the pMAGic 7.1 vector to produce four shRNA vectors: CLU-1, CLU-2, CLU-3, and CLU-4 (19). Transfection of cells was performed using GenJet DNA in vitro transfection reagent (SignaGen Laboratories, Rockville, MD, USA). pCDNA3.1-sCLU and its control pCDNA3.1 plasmid were transfected into Bel-7402 and SMMC-7721 cells as previously reported (22).

Statistical analysis. Data are presented as the mean \pm standard deviation (SD) and were analyzed by one-way ANOVA followed by the Dunnett's test with SPSS software (v17.0; SPSS, Inc., Chicago, IL, USA), with values of $\mathrm{P}<0.05$ considered to indicate a statistically significant difference.

\section{Results}

sCLU knockdown decreases HCC cells invasion. In our previous studies, our results showed that meloxicam suppressed HCC cell survival and its cytotoxicity increased in a concentration-dependent manner. Moreover, we found that HCC cells expressed different levels of COX-2 and sCLU protein, and Bel-7402 and SMMC-7721 cells expressed higher levels of COX-2 and sCLU than other HCC cells $(14,19,20)$. Therefore, in the present study, Bel-7402 and SMMC-7721 cells were chosen for the following experiments. CLU has been reported to be associated with invasion and metastasis $(23,24)$. In this study, we first used the shRNA approach to investigate the role of sCLU in HCC cell invasion. In our previous study, we designed four pMAGic7.1-based shRNA vectors (CLU1, CLU2, CLU3, and CLU4) to down-regulate expression of sCLU in HCC cell 

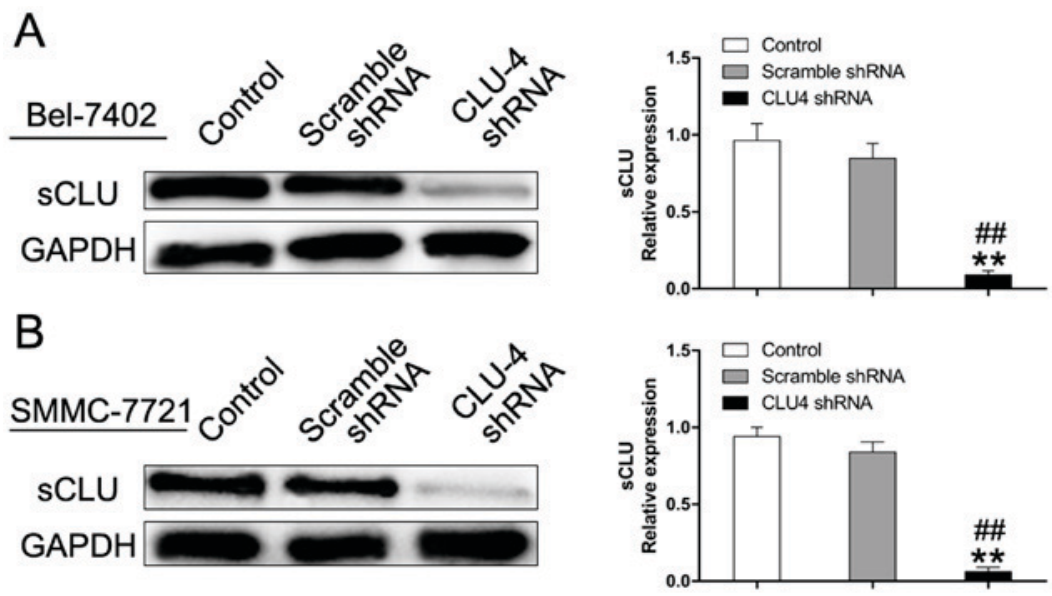

C
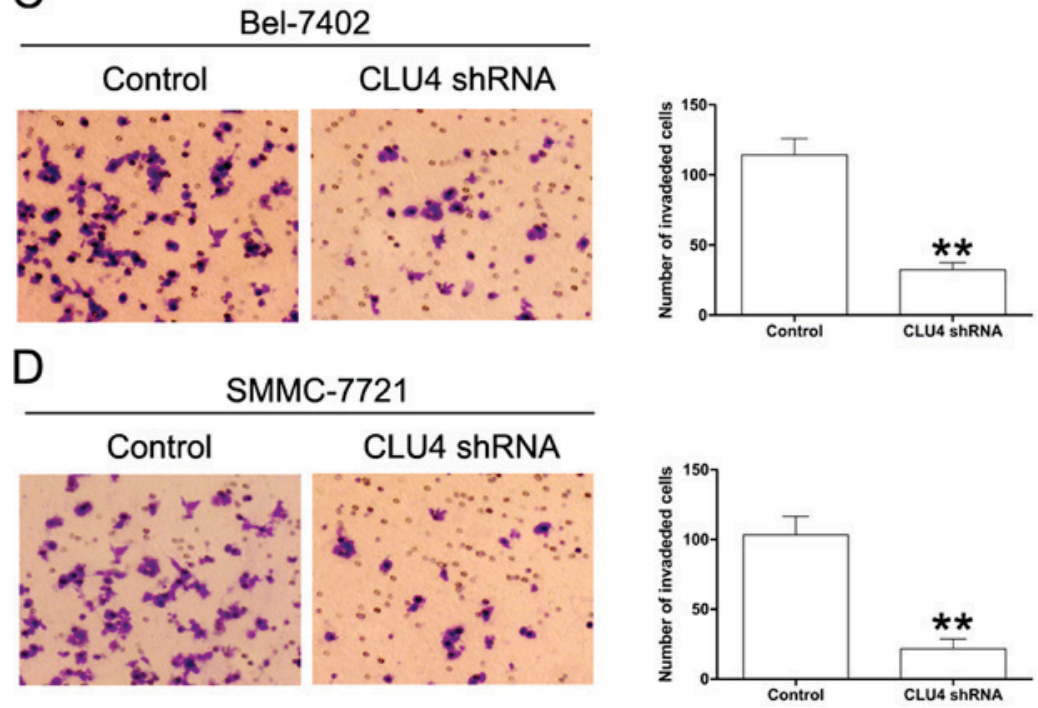

Figure 1. Effect of sCLU knockdown on the invasive behavior of Bel-7402 and SMMC-7721 cells. (A and B) Bel-7402 or SMMC-7721 cells (control), or the cells transfected with scramble shRNA or CLU4 shRNA vector, were cultured for $24 \mathrm{~h}$. Cell lysates were harvested and analyzed by western blotting with specific antibodies against sCLU. Levels of GAPDH served as a loading control. ${ }^{* *} \mathrm{P}<0.01$ vs. Control. ${ }^{\# \#} \mathrm{P}<0.01$ vs. scramble shRNA. The data shown are representative of three independent experiments. (C and D) Invasive behavior was analyzed using Matrigel invasion assays after knockdown of sCLU in Bel-7402 and SMMC-7721 cells (magnification, $\mathrm{x} 100$ ). ${ }^{* *} \mathrm{P}<0.01$ vs. Control. Each experiment was performed in triplicate.

lines. We found that CLU4 shRNA displayed the strongest gene-silencing ability (19). Therefore, CLU4 shRNA was used in the current work. As depicted in Fig. 1A and B, CLU4 shRNA significantly decreased expression of sCLU. Matrigel invasion assays showed that knockdown of sCLU by CLU4 shRNA notably impaired invasive abilities of both Bel-7402 and SMMC-7721 cells suggesting the essential role of sCLU in conferring invasive properties to HCC cells (Fig. 1C and D).

sCLU over-expression increases HCC cancer cell invasion. To further investigate the effect of sCLU in regulating Bel-7402 and SMMC-7721 cell invasion, sCLU was over-expressed (Fig. 2A). As shown in Fig. 2, over-expression of sCLU significantly enhanced invasive abilities of both Bel-7402 and SMMC-7721 cells. These results supported our hypothesis that sCLU confers invasive characteristics to Bel-7402 and SMMC-7721 cells.

$s C L U$ regulates expression of MMP-2 and E-cadherin in HCC cells in vitro. As matrix metallo-proteinase (MMP)-2 and E-cadherin activity has been considered to exert a crucial role in tumor invasion, we first examined whether sCLU could lead to MMP-2 and E-cadherin activity in Bel-7402 and SMMC-7721 cells. As shown in Fig. 3A and B, cells transfected with CLU4 shRNA significantly suppressed expression of MMP-2 and enhanced the extent of E-cadherin. Furthermore, we examined the effect of sCLU over-expression on expression of MMP-2 and E-cadherin. As expected, Bel-7402 and SMMC-7721 cells transfected with pCDNA3. 1-sCLU notably up-regulated expression of MMP-2 and down-regulated expression of E-cadherin (Fig. 3C and D). These results demonstrated the involvement of sCLU in the regulation of MMP-2 and E-cadherin in HCC cells in vitro.

Meloxicam treatment attenuates HCC cancer cell proliferation and invasion. Our results for the CCK-8 assay presented in Fig. 4A showed that meloxicam notably inhibited proliferation of Bel-7402 and SMMC-7721 cells in a time- and concentration-dependent manner. To further explore the role of meloxicam on invasion of HCC cancer cells, Bel-7402 and SMMC-7721 cells were treated with meloxicam $(80 \mu \mathrm{M})$ for $24 \mathrm{~h}$. As determined by scratch motility assay, meloxicam treatment significantly suppressed the migratory potential 


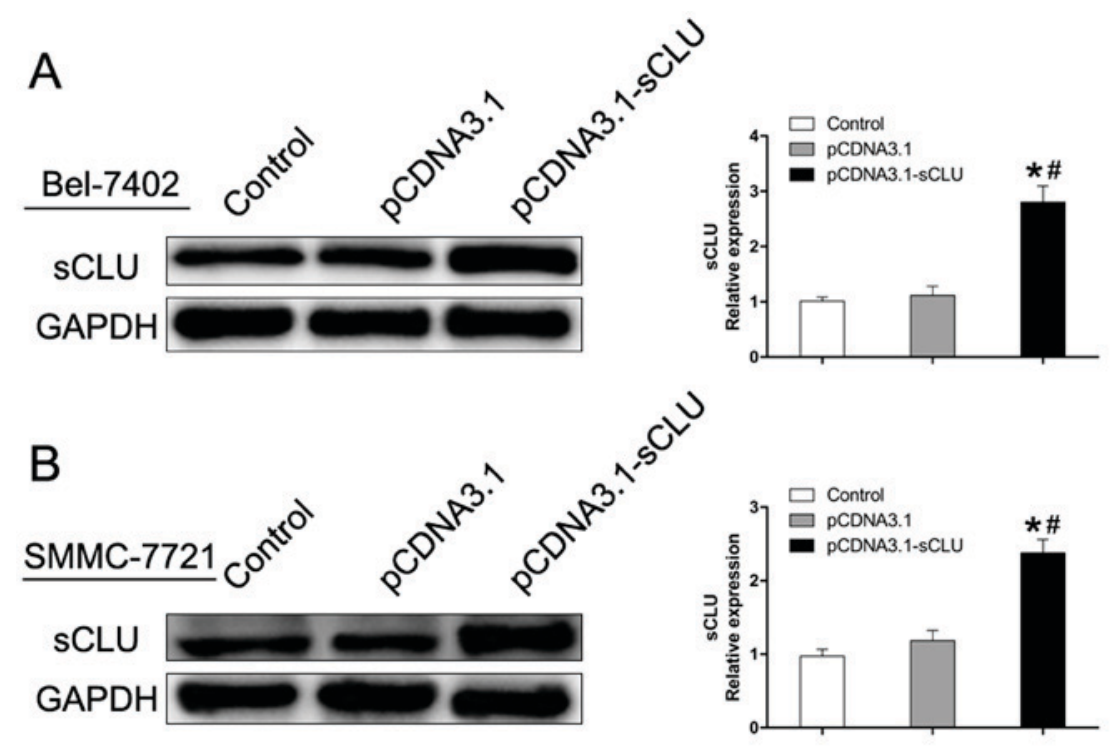

C
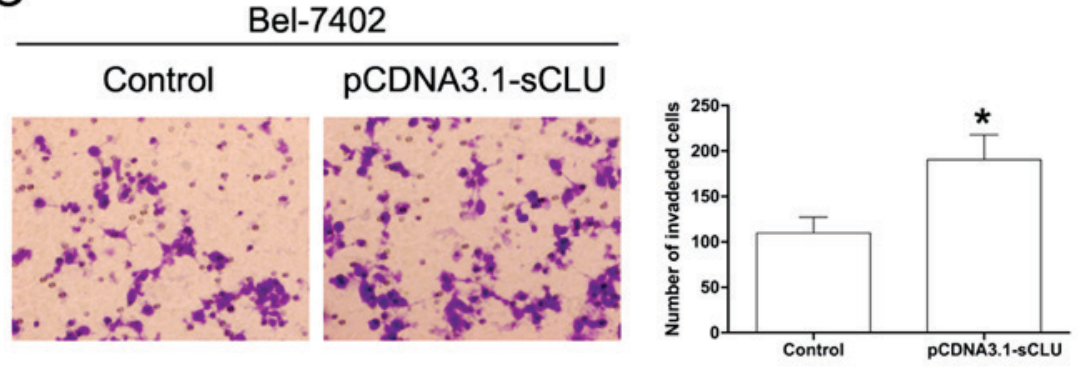

D

SMMC-7721
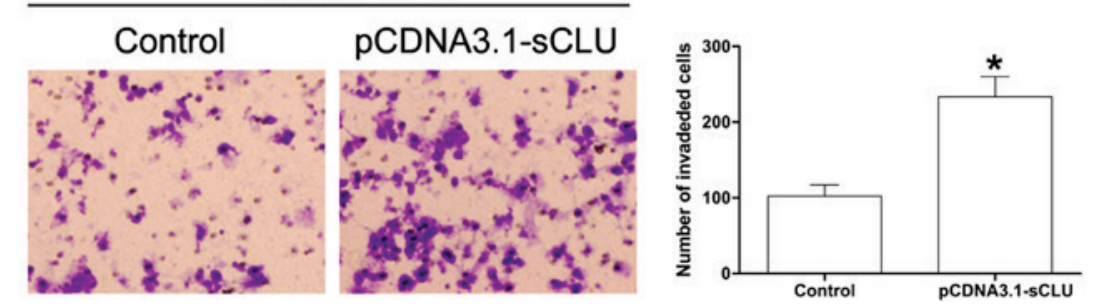

Figure 2. Effect of sCLU over-expression on the invasive capability of Bel-7402 and SMMC-7721 cells. (A and B) Bel-7402 or SMMC-7721 cells (control), or the cells transfected with pCDNA3.1 or pCDNA3.1-sCLU, were cultured for $24 \mathrm{~h}$. Cell lysates were harvested and analyzed by western blotting with specific antibodies against sCLU. Levels of GAPDH served as a loading control. ${ }^{*} \mathrm{P}<0.05$ vs. Control. ${ }^{*} \mathrm{P}<0.05$ vs. pCDNA3.1-sCLU. The data shown are representative of three independent experiments. (C and D) Invasive behavior was analyzed using Matrigel invasion assays after over-expression of sCLU in Bel-7402 and SMMC-7721 cells (magnification, $\mathrm{x} 100$ ). " $\mathrm{P}<0.05$ vs. Control. Each experiment was performed in triplicate.

of Bel-7402 and SMMC-7721 cells (Fig. 4B). Furthermore, we used Transwell assays to examine invasion. As shown in Fig. 4C, the results were consistent with those of the scratch assay. Considering the crucial role of sCLU in invasion, we hypothesized whether meloxicam could target sCLU for its anti-tumor effect. To clarify this issue, we examined expression of sCLU in meloxicam treatment in Bel-7402 and SMMC-7721 cells. As shown in Fig. 4D, meloxicam treatment significantly decreased expression of sCLU in a dose-dependent manner in Bel-7402 and SMMC-7721 cells. These data suggested that meloxicam is an effective inhibitor against invasion and regulates sCLU in HCC cells.

sCLU is responsible for meloxicam-regulated suppression of proliferation and invasion in HCC cells. To investigate whether
sCLU contributes to the function of meloxicam in mediating proliferation and invasion in Bel-7402 and SMMC-7721 cells, expression of sCLU was knocked down by CLU4 shRNA. As shown in Fig. 5A, exposure to meloxicam after inhibition of sCLU induced a significant suppression of cell proliferation. Moreover, we found that suppressed expression of sCLU (Fig. 5B) dramatically reduced invasion in Bel-7402 and SMMC-7721 cells treated with meloxicam (Fig. 5C and D). These data revealed that sCLU is an important mediator for meloxicam in mediating cell proliferation and invasion in Bel-7402 and SMMC-7721 cells.

Up-regulation of MMP-2 by sCLU is dependent on AKT activation in HCC cells in vitro. The AKT pathway has been considered to exert a crucial role in regulating cell 
A Bel-7402
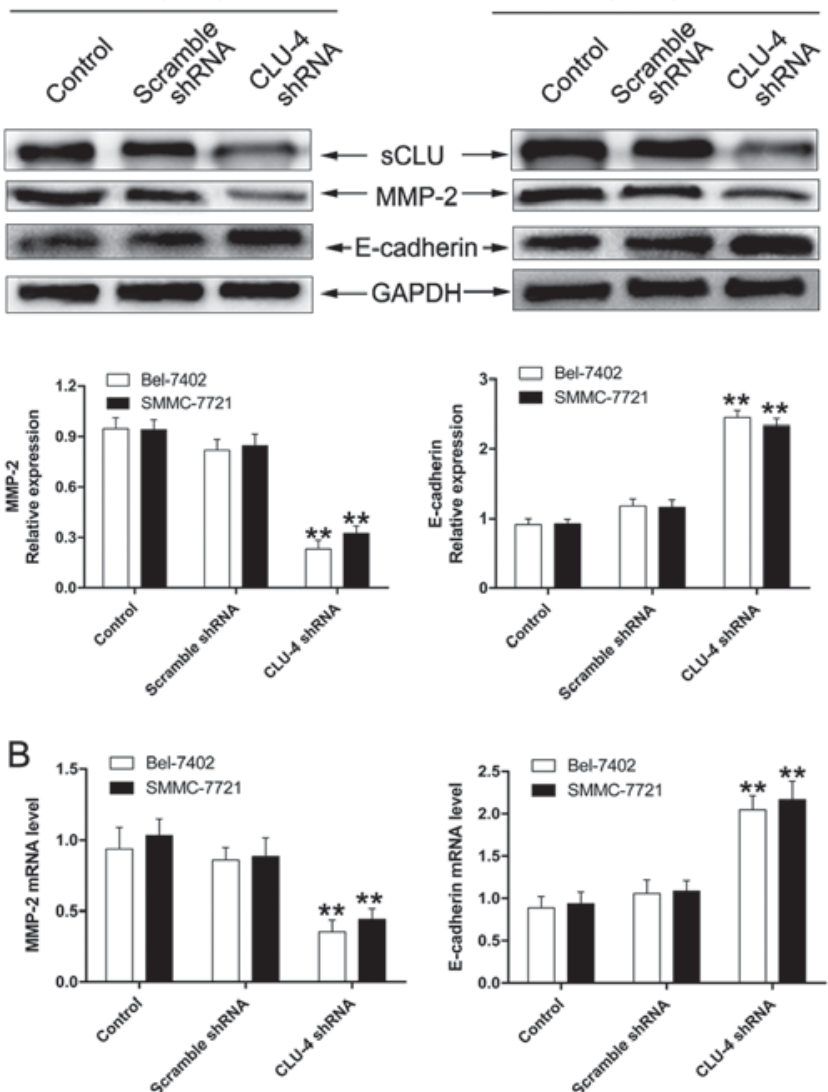
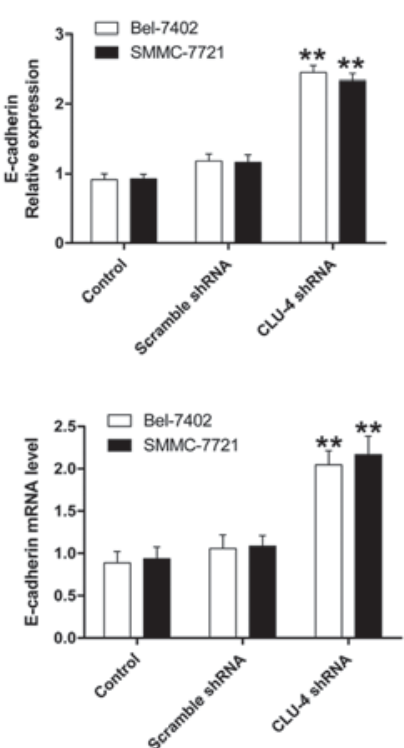

C

Bel-7402
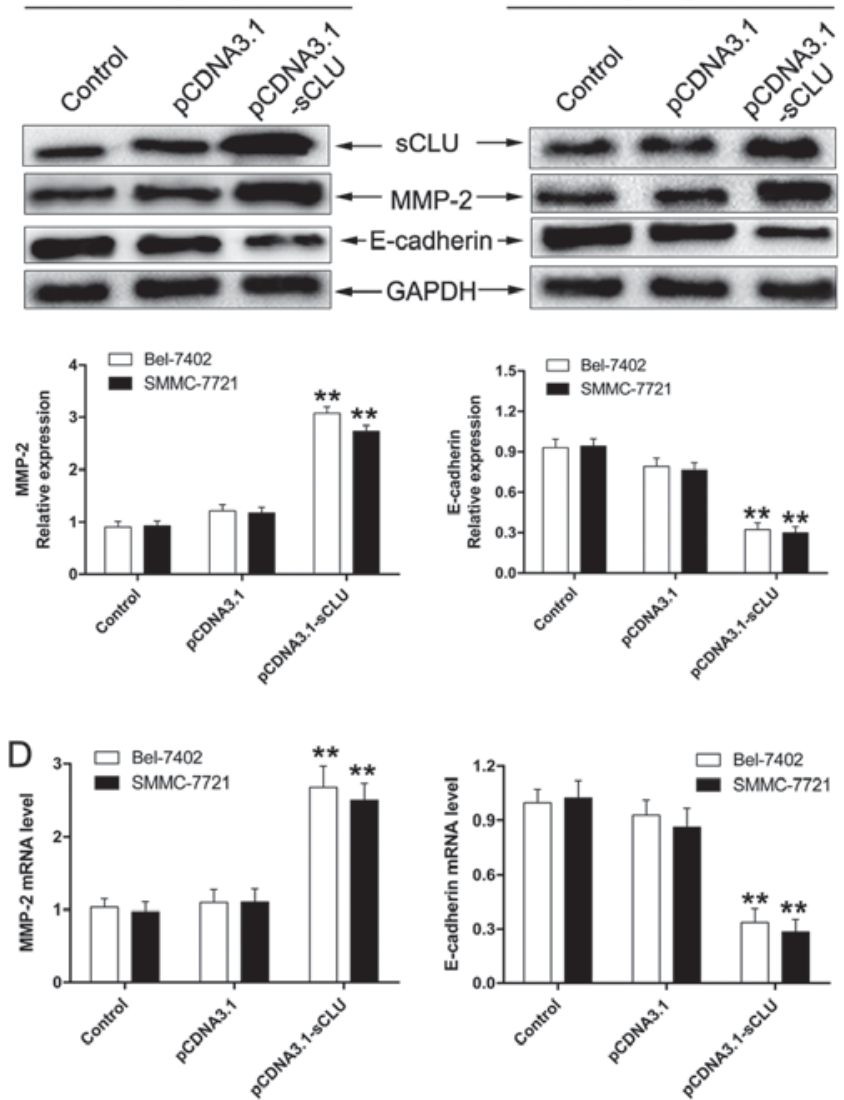

Figure 3. Effect of sCLU on expression of MMP-2 and E-cadherin in HCC cells in vitro. (A and B) Bel-7402 or SMMC-7721 cells (control), or the cells transfected with scramble shRNA or CLU4 shRNA vector, were cultured for $24 \mathrm{~h}$. Cell lysates were harvested and analyzed by western blotting with specific antibodies against MMP-2 and E-cadherin. mRNA expression of MMP-2 and E-cadherin was determined by real-time quantitative RT-PCR. GAPDH served as an internal control. ${ }^{* *} \mathrm{P}<0.01$ vs. control. (C and D) Cells transfected with pCDNA3.1 or pCDNA3.1-sCLU were cultured for $24 \mathrm{~h}$. Then the cell lysates were harvested and analyzed by western blotting with specific antibodies against MMP-2 and E-cadherin. mRNA expression of MMP-2 and E-cadherin was determined by real-time quantitative RT-PCR. GAPDH served as an internal control. Levels of GAPDH served as a loading control. ${ }^{* *} \mathrm{P}<0.01 \mathrm{vs}$. control. The data shown are representative of three independent experiments.

growth, proliferation, survival, and motility (25-28). Our previous studies also revealed that sCLU suppressed HCC cell apoptosis induced by AKT inhibition (19). In this study, to investigate whether the AKT signaling pathway mediates the regulatory effect of sCLU on MMP-2 expression, Bel-7402 and SMMC-7721 cells were pre-incubated with MK-2206, an AKT specific inhibitor. As shown in Fig. 6A and B, the extent of p-AKT was decreased in both cells after transfection with CLU4 shRNA. However, over-expression of sCLU significantly enhanced the level of p-AKT in Bel-7402 and SMMC-7721 cells. Furthermore, expression of MMP-2 was significantly inhibited by MK-2206 (Fig. 6C and D). In addition, suppression of AKT by MK-2206 significantly reduced invasion of Bel-7402 and SMMC-7721 cells transfected by pCDNA3.1-sCLU (Fig. 6E). These results suggested that the AKT signaling pathway may exert an important role in mediating MMP-2 expression and sCLU-induced invasiveness in Bel-7402 and SMMC-7721 cells.

\section{Discussion}

Recurrence and metastasis are recognized as the major leading causes of poor prognosis of HCC patients (23). In spite of great progress in exploring the molecular mechanism of invasion and metastasis of $\mathrm{HCC}$, it has fallen well short of its goals due to the poor prognosis of HCC patients. Therefore, it is worthwhile to study the molecular mechanism of HCC invasiveness.

sCLU has been considered as a stress-induced chaperone that confers proliferative and survival advantages to many cancers, including retinal (29), breast (30), lung (22), ovarian (24), and cervical (31) cancer. Our previous studies also demonstrated that sCLU is over-expressed in HCC cells $(19,20)$. Recently, several studies reported that sCLU over-expression plays an important role in regulating invasion and migration $(23,32)$. However, the role of sCLU in HCC cell invasion has yet to be elucidated. In this study, we observed that down-regulation of sCLU by CLU4 shRNA significantly alleviated invasiveness whereas over-expression sCLU notably enhanced the number of invasive cells in Bel-7402 and SMMC-7721 cells. Furthermore, we found that sCLU exerted a crucial role in regulating invasiveness of Bel-7402 and SMMC-7721 cells via mediating the levels of MMP-2 and E-cadherin. These results suggested that sCLU confers invasive characteristics to HCC cells through regulating expression of MMP-2 and E-cadherin protein in HCC cells.

The selective COX-2 inhibitor meloxicam has been reported to exert anti-invasion responses in various 
A

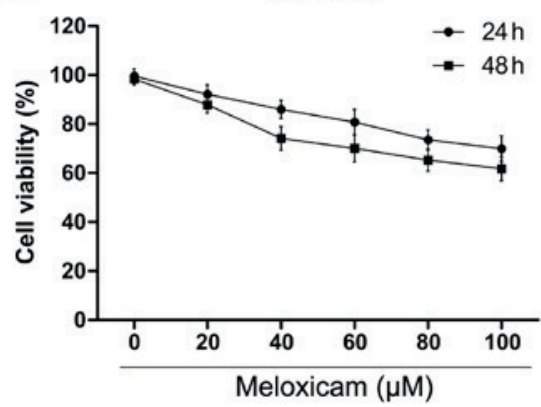

B
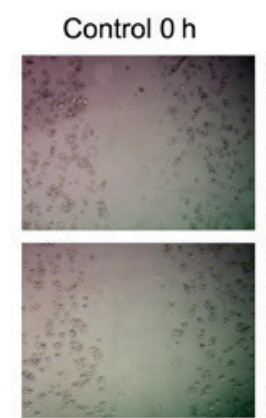

C

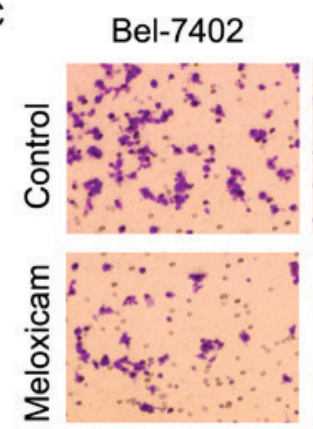

Meloxicam $\mathrm{Oh}$
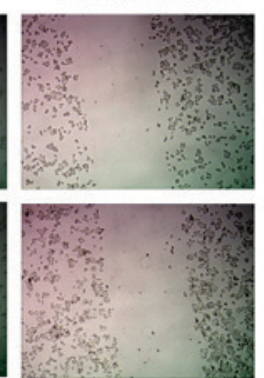

SMMC-7721

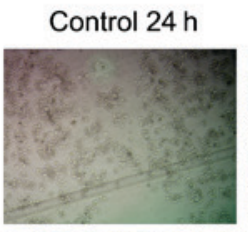

Meloxicam $24 \mathrm{~h}$
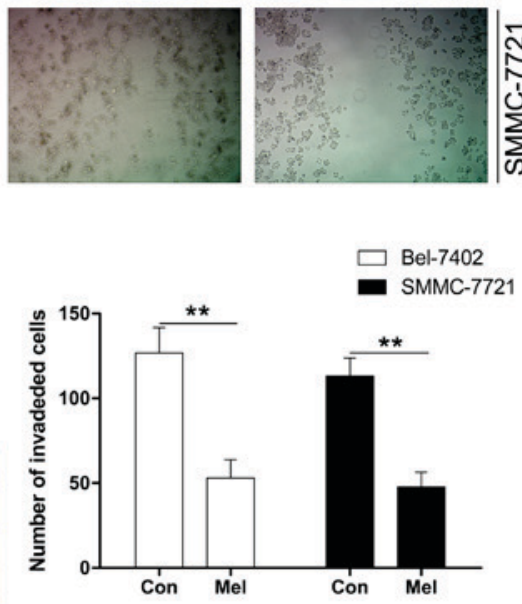

D
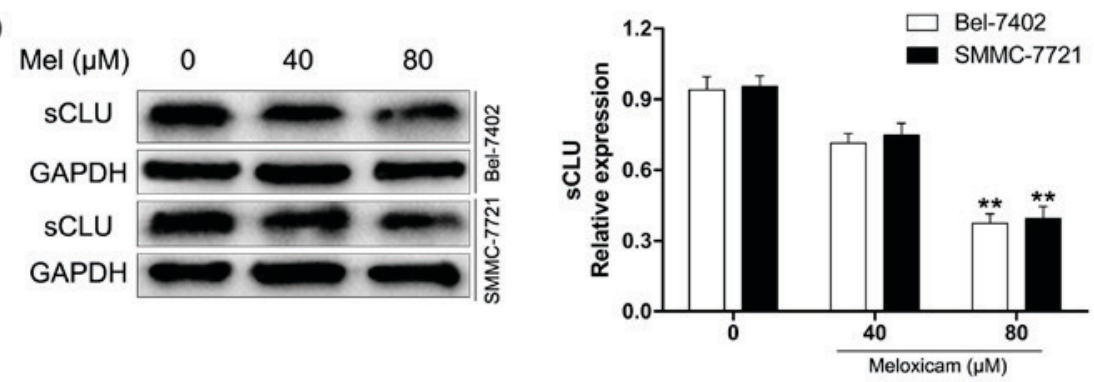

Figure 4. Meloxicam treatment blocks HCC cell proliferation and invasion. (A) Bel-7402 and SMMC-7721 cells were incubated with meloxicam in various concentrations $(0$ to $100 \mu \mathrm{M})$ for 24 or $48 \mathrm{~h}$. Cell vitality was assessed by the CCK- 8 assay. The data shown are representative of three independent experiments. (B) Meloxicam ( $80 \mu \mathrm{M})$ suppressed motility in a scratch assay of Bel-7402 and SMMC-7721 cells. Cell motility was quantified by measuring the distance between the migrating cell boundaries. (C) Representative images were captured from Bel-7402 and SMMC-7721 cells incubated with meloxicam $(80 \mu \mathrm{M})$ or not and subjected to cell invasion assays as described in Materials and methods (magnification, $\mathrm{x} 100)$. ${ }^{* *} \mathrm{P}<0.01$. (D) Cells were exposed to the control (untreated) or meloxicam $(80 \mu \mathrm{M})$ for $24 \mathrm{~h}$. Cell lysates were harvested and analyzed by western blotting with specific antibodies against sCLU. Levels of GAPDH served as a loading control. ${ }^{* *} \mathrm{P}<0.01$ vs. control (untreated). The data shown are representative of three independent experiments.

tumors (33-35). Our previous data also showed that meloxicam inhibited migration and invasion in $\mathrm{HCC}$ cells $(14,15)$. However, it remains unknown whether sCLU participates in the anti-invasion effects of HCC to meloxicam. In this current work, we found that the extent of sCLU was decreased after treatment with $80 \mu \mathrm{M}$ meloxicam. These results might contribute to the observation that meloxicam could suppress the proliferation and invasion of Bel-7402 and SMMC-7721 cells. Moreover, the combinations of meloxicam and CLU4 shRNA significantly decreased invasion in Bel-7402 and SMMC-7721 cells. These finding suggested that as an important mediator of invasiveness, sCLU may be responsible for meloxicam regulated suppression of proliferation and invasion in HCC cells.

In the present study, we also investigated whether the AKT signaling pathway was involved in regulation of MMP-2 expression by treating Bel-7402 and SMMC-7721 cells with the AKT specific inhibitor, MK-2206. We found that inhibition of AKT by MK-2206 dramatically decreased the level of MMP-2 in over-expressing sCLU HCC cells. Several studies reported 
A

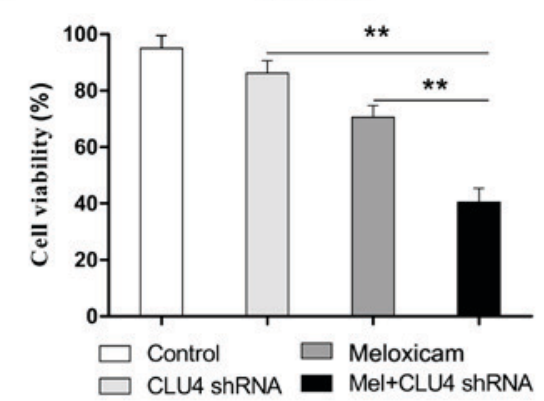

B

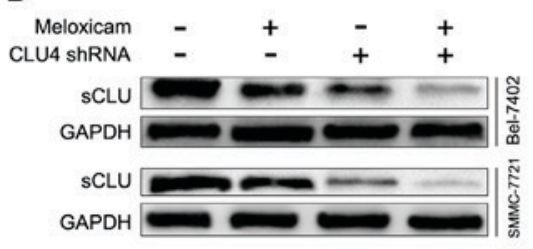

C Control

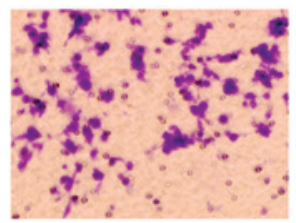

Meloxicam
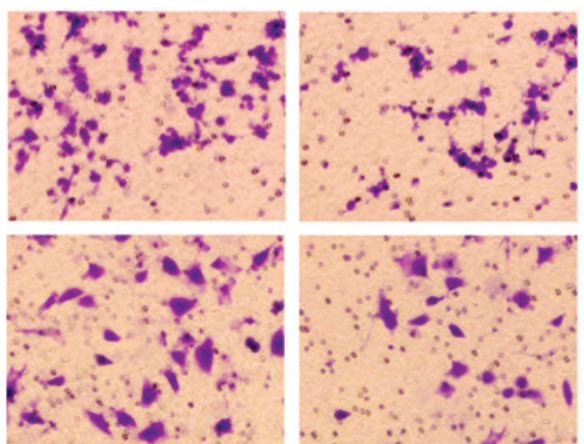

D

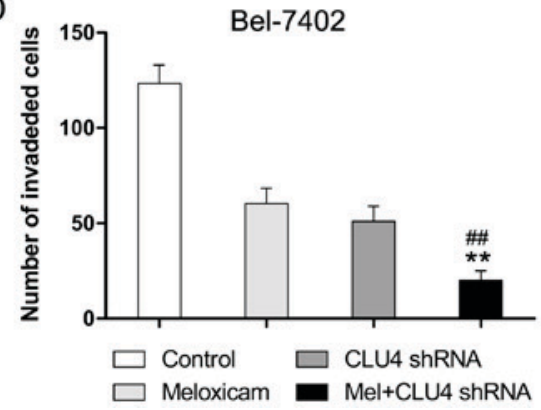

SMMC-7721

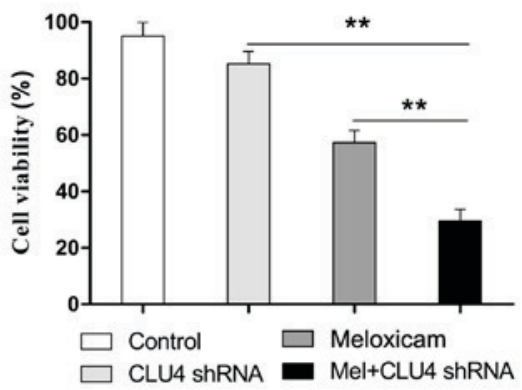

Bel-7402
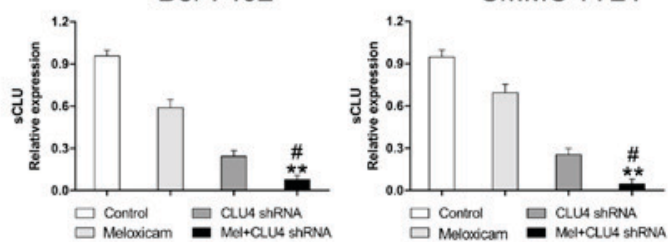

CLU4 shRNA Mel+CLU4 shRNA
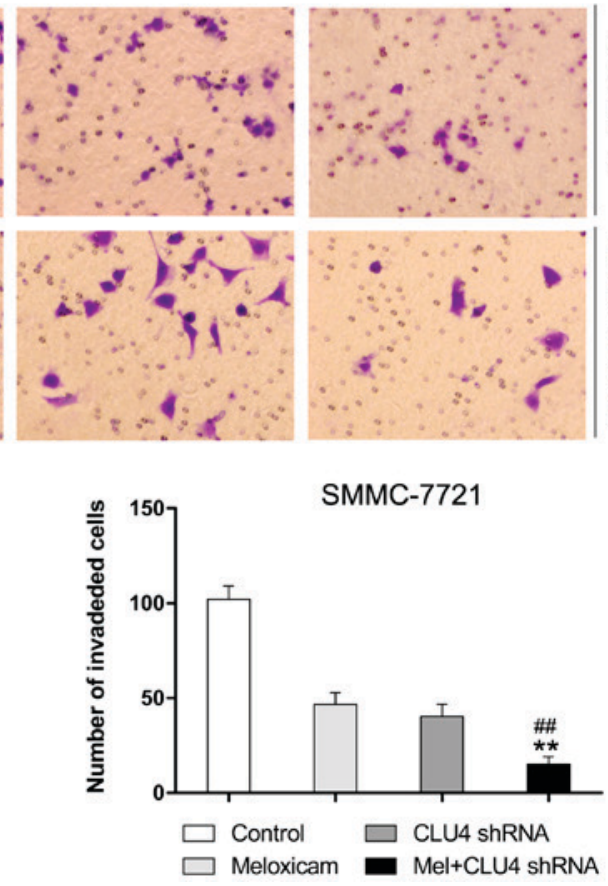

Figure 5. sCLU is responsible for meloxicam-mediated induction of proliferation and invasion in HCC cells in vitro. (A) Relative cell proliferation rate in Bel-7402 and SMMC-7721 cells after treatment of meloxicam, sCLU knockdown, and a combination. Cell vitality was assessed by the CCK-8 assay. The data shown are representative of three independent experiments. ${ }^{* *} \mathrm{P}<0.01$. (B) The protein level of sCLU after the treatment of meloxicam, sCLU knockdown, and a combination. Levels of GAPDH served as a loading control. ${ }^{* *} \mathrm{P}<0.01$ vs. meloxicam. ${ }^{*} \mathrm{P}<0.05$ vs. CLU4 shRNA. The data shown are representative of three independent experiments. (C and D) Representative images of the invasion assay in Bel-7402 and SMMC-7721 cells after treatment of meloxicam, sCLU knockdown, and a combination. (magnification, $\mathrm{x} 100$ ). ${ }^{* *} \mathrm{P}<0.01$ vs. meloxicam. ${ }^{\# \#} \mathrm{P}<0.01$ vs. CLU4 shRNA. The data shown are representative of three independent experiments.

that CLU confers proliferative and survival advantages through the AKT signaling pathway $(23,36)$. In the present study, we found that over-expression of sCLU significantly potentiated expression of p-AKT and MMP-2. However, down-regulation of sCLU by CLU4 shRNA alleviated the extent of p-AKT. These data revealed that sCLU may promote HCC invasion via the AKT signaling pathway.

In conclusion, we found that the inhibitory effect of meloxicam on invasion in HCC cells was through down-regulation of sCLU expression. Furthermore, our data promote a novel mechanism that sCLU activates the AKT signaling pathway, which promotes expression of MMP-2 and induces invasion of HCC cells. The targeting of sCLU suggests a novel therapeutic strategy against invasion in HCC.

\section{Acknowledgements}

Thanks to Dr. Edward C. Mignot, Shandong University, for linguistic advice. 
A

Bel-7402
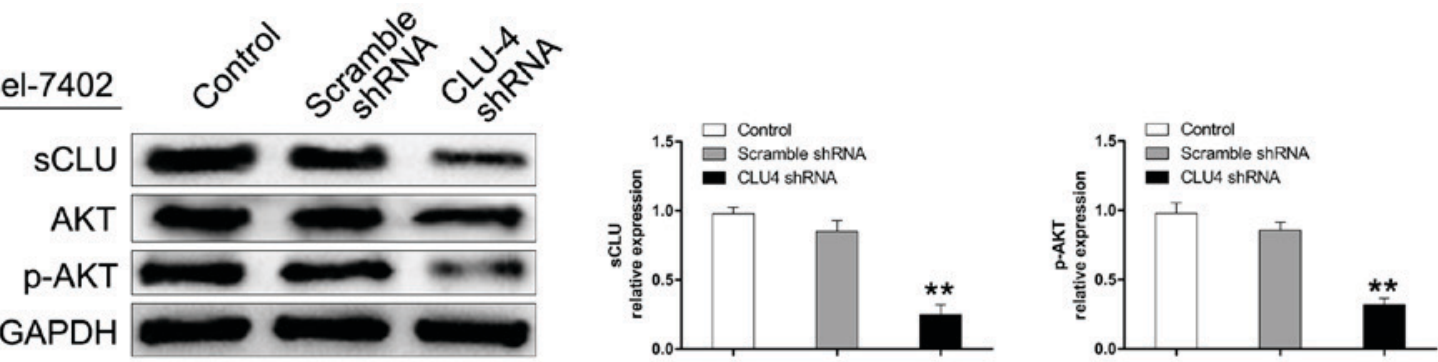

B
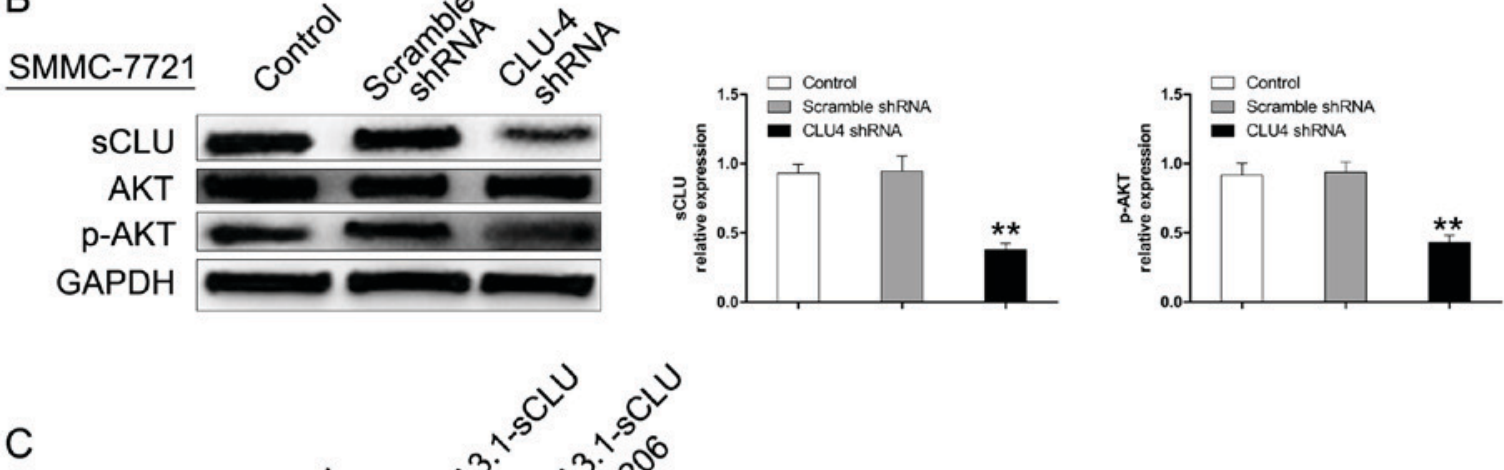

C

Bel-7402

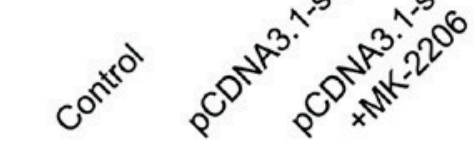

AKT

p-AKT

MMP-2

GAPDH
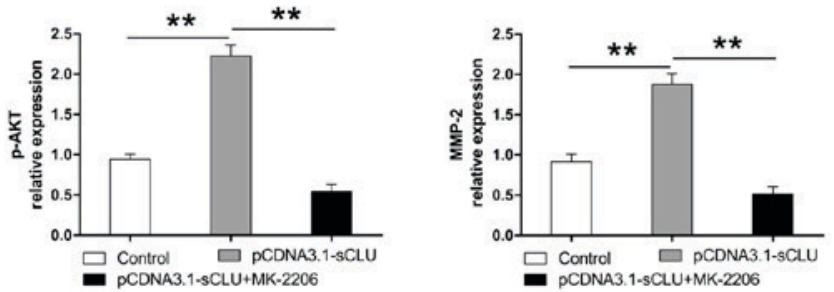

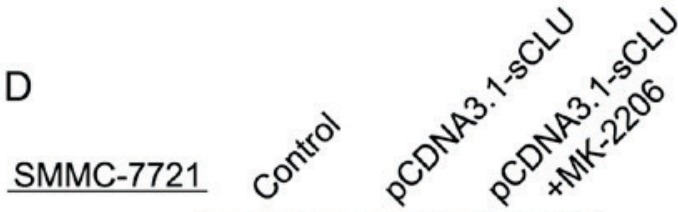
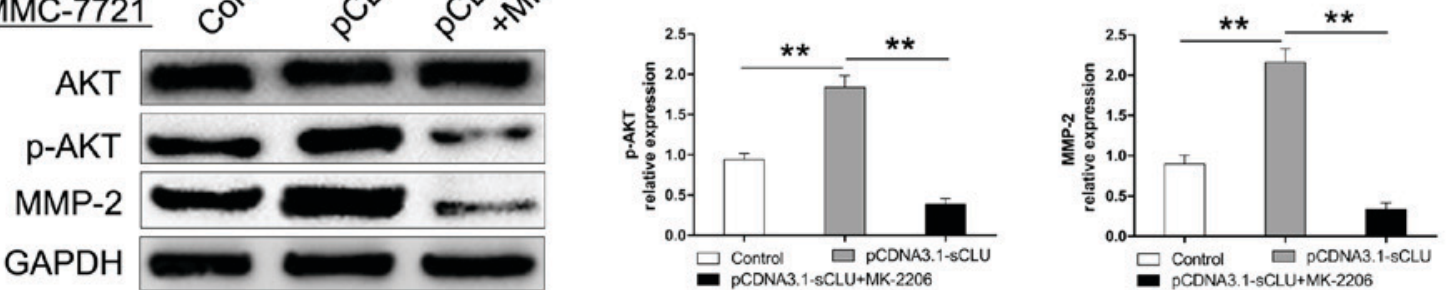

E

pCDNA3.1-sCLU pCDNA3.1-sCLU+MK-2206
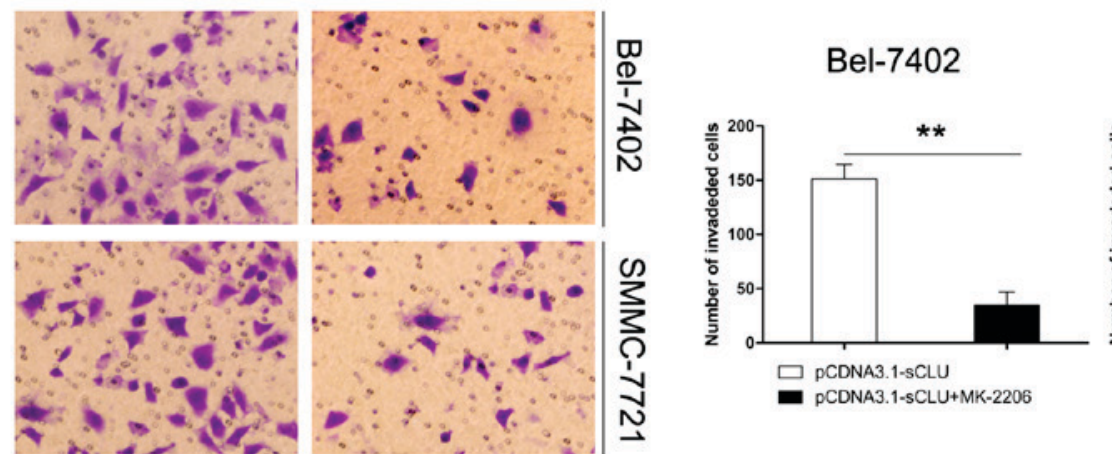

SMMC-7721

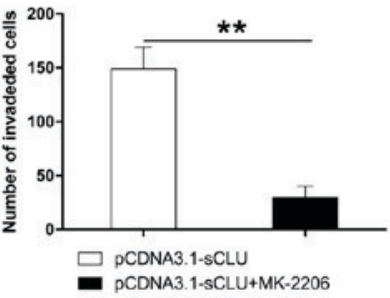

Figure 6. sCLU enhances MMP-2 expression via AKT activation in HCC cells in vitro. (A and B) Bel-7402 or SMMC-7721 cells (control), or cells transfected with scramble shRNA or CLU4 shRNA vector, were cultured for $24 \mathrm{~h}$. Levels of sCLU, AKT, and p-AKT were detected by western blotting. Levels of GAPDH served as a loading control. ${ }^{* *} \mathrm{P}<0.01 \mathrm{vs}$. control. (C and D) Cells were treated with $5 \mu \mathrm{M}$ MK-2206 for $24 \mathrm{~h}$. Levels of p-Akt, Akt, and MMP-2 were detected by western blotting. Levels of GAPDH served as a loading control. ${ }^{* *} \mathrm{P}<0.01$. (E) Invasion assays were done for the HCC cells following treatment with $5 \mu \mathrm{M}$ MK-2206 for $24 \mathrm{~h}$ (magnification, $\mathrm{x} 100){ }^{* * *} \mathrm{P}<0.01$. Each experiment was performed in triplicate. 


\section{References}

1. Jemal A, Bray F, Center MM, Ferlay J, Ward E and Forman D: Global cancer statistics. CA Cancer J Clin 61: 69-90, 2011.

2. Torre LA, Bray F, Siegel RL, Ferlay J, Lortet-Tieulent J and Jemal A: Global cancer statistics, 2012. CA Cancer J Clin 65: 87-108, 2015.

3. Bruix J, Gores GJ and Mazzaferro V: Hepatocellular carcinoma: Clinical frontiers and perspectives. Gut 63: 844-855, 2014.

4. Said A and Wells J: Management of hepatocellular carcinoma. Minerva Med 100: 51-68, 2009.

5. Zhuang L, Xu L, Wang P, Jiang Y, Yong P, Zhang C, Zhang H, Meng $Z$ and Yang P: Na+/K+-ATPase $\alpha 1$ subunit, a nove therapeutic target for hepatocellular carcinoma. Oncotarget 6 : 28183-28193, 2015.

6. Yu SM and Kim SJ: DNA-hypomethylating agent, 5'-azacytidine, induces cyclooxygenase-2 expression via the PI3-kinase/Akt and extracellular signal-regulated kinase-1/2 pathways in human HT1080 fibrosarcoma cells. Int J Oncol 47: 1469-1475, 2015.

7. Diab S, Fidanzi C, Léger DY, Ghezali L, Millot M, Martin F, Azar R, Esseily F, Saab A, Sol V, et al: Berberis libanotica extract targets $\mathrm{NF}-\kappa \mathrm{B} / \mathrm{COX}-2, \mathrm{PI} 3 \mathrm{~K} / \mathrm{Akt}$ and mitochondrial/caspase signalling to induce human erythroleukemia cell apoptosis. Int J Oncol 47: 220-230, 2015.

8. Cao J, Guo T, Dong Q, Zhang J and Li Y: miR-26b is downregulated in human tongue squamous cell carcinoma and regulates cell proliferation and metastasis through a COX-2-dependent mechanism. Oncol Rep 33: 974-980, 2015.

9. Wang L, Wang Z, Li J,Zhang W, Ren F and Yue W: NFATc1 activation promotes the invasion of U251 human glioblastoma multiforme cells through COX-2. Int J Mol Med 35: 1333-1340, 2015.

10. Liu M, Li CM, Chen ZF, Ji R, Guo QH, Li Q, Zhang HL and Zhou YN: Celecoxib regulates apoptosis and autophagy via the PI3K/Akt signaling pathway in SGC-7901 gastric cancer cells Int J Mol Med 33: 1451-1458, 2014.

11. Yusup G, Akutsu Y, Mutallip M, Qin W, Hu X, Komatsu-Akimoto A, Hoshino I, Hanari N, Mori M, Akanuma N, et al: A COX-2 inhibitor enhances the antitumor effects of chemotherapy and radiotherapy for esophageal squamous cell carcinoma. Int J Oncol 44: 1146-1152, 2014.

12. Qian M, Qian D, Jing H, Li Y, Ma C and Zhou Y: Combined cetuximab and celecoxib treatment exhibits a synergistic anticancer effect on human oral squamous cell carcinoma in vitro and in vivo. Oncol Rep 32: 1681-1688, 2014.

13. Li J, Chen X, Dong X, Xu Z, Jiang H and Sun X: Specific COX-2 inhibitor, meloxicam, suppresses proliferation and induces apoptosis in human HepG2 hepatocellular carcinoma cells. J Gastroenterol Hepatol 21: 1814-1820, 2006.

14. Dong X, Li R, Xiu P, Dong X, Xu Z, Zhai B, Liu F, Jiang H, Sun X, Li J and Qiao H: Meloxicam executes its antitumor effects against hepatocellular carcinoma in COX-2-dependent and -independent pathways. PLoS One 9: e92864, 2014.

15. Zhong J, Xiu P, Dong X, Wang F, Wei H, Wang X, Xu Z, Liu F, Li T, Wang Y and Li J: Meloxicam combined with sorafenib synergistically inhibits tumor growth of human hepatocellular carcinoma cells via ER stress-related apoptosis. Oncol Rep 34: 2142-2150, 2015.

16. Zhong J, Dong X, Xiu P, Wang F, Liu J, Wei H, Xu Z, Liu F, Li T and Li J: Blocking autophagy enhances meloxicam lethality to hepatocellular carcinoma by promotion of endoplasmic reticulum stress. Cell Prolif 48: 691-704, 2015.

17. Shannan B, Seifert M, Leskov K, Willis J, Boothman D, Tilgen W and Reichrath J: Challenge and promise: Roles for clusterin in pathogenesis, progression and therapy of cancer. Cell Death Differ 13: 12-19, 2006.

18. Chen Q, Wang Z, Zhang K, Liu X, Cao W, Zhang L, Zhang S, Yan B, Wang Y and Xia C: Clusterin confers gemcitabine resistance in pancreatic cancer. World J Surg Oncol 9: 59, 2011.

19. Xiu P, Dong X, Dong X, Xu Z, Zhu H, Liu F, Wei Z, Zhai B, Kanwar JR, Jiang H, et al: Secretory clusterin contributes to oxaliplatin resistance by activating Akt pathway in hepatocellular carcinoma. Cancer Sci 104: 375-382, 2013.

20. Xiu P, Xu Z, Liu F, Li Z, Li T, Zou F, Sun X and Li J: Downregulating sCLU enhances the sensitivity of hepatocellular carcinoma cells to gemcitabine by activating the intrinsic apoptosis pathway. Dig Dis Sci 59: 1798-1809, 2014.
21. Wang F, Dong X, Xiu P, Zhong J, Wei H, Xu Z, Li T, Liu F, Sun X and Li J: T7 peptide inhibits angiogenesis via downregulation of angiopoietin-2 and autophagy. Oncol Rep 33: 675-684, 2015.

22. Zhang B, Zhang K, Liu Z, Hao F, Wang M, Li X, Yin Z and Liang H: Secreted clusterin gene silencing enhances chemosensitivity of a549 cells to cisplatin through AKT and ERK1/2 pathways in vitro. Cell Physiol Biochem 33: 1162-1175, 2014.

23. Wang C, Jin G, Jin H, Wang N, Luo Q, Zhang Y, Gao D, Jiang K, Gu D, Shen Q, et al: Clusterin facilitates metastasis by EIF3I/Akt/MMP13 signaling in hepatocellular carcinoma. Oncotarget 6: 2903-2916, 2015.

24. Fu Y, Lai Y, Liu J, Liu X, You Z and Yang G: Lentivirus-mediated shRNA interference of clusterin blocks proliferation, motility, invasion and cell cycle in the ovarian cancer cells. J Ovarian Res 8: 59, 2015.

25. He J, Zhu G, Gao L, Chen P, Long Y, Liao S, Yi H, Yi W, Pei Z, $\mathrm{Wu} \mathrm{M}$, et al: Fra-1 is upregulated in gastric cancer tissues and affects the PI3K/Akt and p53 signaling pathway in gastric cancer. Int J Oncol 47: 1725-1734, 2015.

26. Raha S, Yumnam S, Hong GE, Lee HJ, Saralamma VV, Park HS, Heo JD, Lee SJ, Kim EH, Kim JA and Kim GS: Naringin induces autophagy-mediated growth inhibition by downregulating the PI3K/Akt/mTOR cascade via activation of MAPK pathways in AGS cancer cells. Int J Oncol 47: 1061-1069, 2015.

27. Jin H, Qiao F, Wang Y, Xu Y and Shang Y: Curcumin inhibits cell proliferation and induces apoptosis of human non-small cell lung cancer cells through the upregulation of miR-192-5p and suppression of PI3K/Akt signaling pathway. Oncol Rep 34: 2782-2789, 2015.

28. Lim HS, Kang YJ, Sung B, Kim SH, Kim MJ, Kim HR, Kim SJ, Choi YH, Moon HR, Chung HY and Kim ND: Novel dihydrobenzofuro[4,5-b][1,8]naphthyridin-6-one derivative, MHY-449, induces cell cycle arrest and apoptosis via the downregulation of Akt in human lung cancer cells. Oncol Rep 34: 2431-2438, 2015.

29. Song HB, Jun HO, Kim JH, Yu YS, Kim KW, Min BH and Kim JH: Anti-apoptotic effect of clusterin on cisplatin-induced cell death of retinoblastoma cells. Oncol Rep 30: 2713-2718, 2013.

30. Wang Y, Wang X, Zhao H, Liang B and Du Q: Clusterin confers resistance to TNF-alpha-induced apoptosis in breast cancer cells through NF-kappaB activation and Bcl-2 overexpression. J Chemother 24: 348-357, 2012

31. Lee JH, Lee JY, Rho SB, Choi JS, Lee DG, An S, Oh T, Choi DC and Lee SH: PACAP inhibits tumor growth and interferes with clusterin in cervical carcinomas. FEBS Lett 588: 4730-4739, 2014.

32. Hwang S, Lee DH, Lee IK, Park YM and Jo I: Far-infrared radiation inhibits proliferation, migration, and angiogenesis of human umbilical vein endothelial cells by suppressing secretory clusterin levels. Cancer Lett 346: 74-83, 2014.

33. Qiu X, Cheng JC, Chang HM and Leung PC: COX2 and PGE2 mediate EGF-induced E-cadherin-independent human ovarian cancer cell invasion. Endocr Relat Cancer 21: 533-543, 2014.

34. Bhattacharya A, Li Y, Shi Y and Zhang Y: Enhanced inhibition of urinary bladder cancer growth and muscle invasion by allyl isothiocyanate and celecoxib in combination. Carcinogenesis 34 : 2593-2599, 2013

35. Chen Z, Liu M, Liu X, Huang S, Li L, Song B, Li H, Ren Q, Hu Z, Zhou Y and Qiao L: COX-2 regulates E-cadherin expression through the NF- $\kappa \mathrm{B} /$ Snail signaling pathway in gastric cancer. Int J Mol Med 32: 93-100, 2013.

36. Ammar $\mathrm{H}$ and Closset JL: Clusterin activates survival through the phosphatidylinositol 3-kinase/Akt pathway. J Biol Chem 283: 12851-12861, 2008.

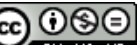

This work is licensed under a Creative Commons Attribution-NonCommercial-NoDerivatives 4.0 International (CC BY-NC-ND 4.0) License. 